\title{
Prevalence of Giardia intestinalis Infection in Household Cats of Tohoku District in Japan
}

\author{
Naoyuki ITOH ${ }^{1)}$, Noboru MURAOKA ${ }^{2)}$, Jun KAWAMATA ${ }^{3)}$, Mikiko AOKI ${ }^{4)}$ and Tadashi ITAGAKI ${ }^{4)}$ \\ ${ }^{1)}$ Kamome Veterinary Clinic, 7-9-2932 Sozen Nishi, Hashikami, Sannohe, Aomori 039-1212, 2) Yokote Animal Hospital, 11 Nagatoro, \\ Inooka, Yokote, Akita 013-0065, ${ }^{3)}$ Chiba Small Animal Clinic, 3-1-18 Noda, Fukushima, Fukushima 960-8055 and ${ }^{4)}$ Laboratory of \\ Veterinary Parasitology, Faculty of Agriculture, Iwate University, 3-18-8 Ueda, Morioka, Iwate 020-8550, Japan
}

(Received 22 August 2005/Accepted 5 October 2005)

\begin{abstract}
Fecal samples obtained from 600 household cats (244 males and 356 females) kept in 3 prefectures of Tohoku district in Japan were examined for Giardia intestinalis antigen, using a commercial enzyme-linked immunosorbent assay (ELISA) kit. G. intestinalis antigen was detected in $40 \%$ of the fecal specimens. The factors such as the age, life style or environmental condition of cats could be significantly related to the positive rate of $G$. intestinalis antigen. In contrast, the investigative district, appearance of feces, sex, breed or origin produced no significant difference in the positive rate. The present results suggest that $G$. intestinalis infection is widely spread in household cats of Tohoku district.
\end{abstract}

KEY WORDS: feline, Giardia intestinalis, prevalence.

J. Vet. Med. Sci. 68(2): 161-163, 2006

The pathogenic protozoa Giardia intestinalis is a ubiquitous parasite of cats and other mammals including humans and cause acute, chronic or intermittent diarrhea in some cases $[6,12,17,18]$. Since the recent molecular biological evidences suggest that some isolates of $G$. intestinalis from cats have zoonotic potential $[1,11,12,18,19]$, a survey on the prevalence of $G$. intestinalis infections in household cats should be needed to prevent zoonotic transmission of the protozoa. However, only a few numbers of studies have been described on the $G$. intestinalis infections in household cats in Japan $[13-15,17]$. The purpose of the present study was to determine the prevalence and epidemiologic background of $G$. intestinalis infections in household cats of Tohoku district in Japan.

Fecal samples were obtained from 600 household cats presented to 3 veterinary practitioners in Aomori $(n=353)$, Akita $(n=188)$ and Fukushima $(n=59)$ Prefectures during the period from April, 2003 to May, 2005. The animals included 244 male and 356 female cats of 11 breeds (554 Japanese domestic cat, 15 American Shorthair, 7 Persian, 6 Scottish Fold, 5 Himalayan, 5 Russian Blue, 3 Abyssinian, 2 Norwegian Forest cat, 1 Siamese cat, 1 Main Coon and 1 American Curl) with the age between 1 month and 22 years old. Fecal specimens were collected immediately after passed, grossly examined for their appearance (normal, soft, diarrhea) and stored at $-20^{\circ} \mathrm{C}$ until analysis. G. intestinalisspecific antigen in fecal samples was detected by enzymelinked immunosorbent assay (ELISA) using a commercially available kit (RIDASCREEN ${ }^{\circledR}$ Giardia, R-Biopharm AG, Germany) according to the manufacture's instruction. The age (1-6 months old, 7 months -1 year old, 2-5 years old, over 6 years old), life style (indoors, indoors/outdoors, outdoors), environmental condition (urban, suburban), origin (private cat owner, pet shop/breeding cattery) were also recorded for the cats. The statistical significance of differences in the positive rates of $G$. intestinalis antigen was ana- lyzed by Fisher's exact probability test. A p-value of $<0.05$ was considered significant.

G. intestinalis antigen was detected in $240(40 \%)$ fecal samples of cats from 1 month to 22 years old. Association between the positive rate of $G$. intestinalis antigen and profile data of the animals was shown in Table 1. The positive rate of kitten from 1 to 6 months old (49\%) was significantly $(p<0.05)$ higher than that of over 6 years old $(32 \%)$. In addition, the cats kept indoors $(33 \%)$ had a significantly $(p<0.05$ or 0.01 ) lower prevalence than those kept indoors/outdoors $(45 \%)$ or outdoors $(53 \%)$. The environmental condition also related to the positive rate of Giardia: suburban cats $(42 \%)$ had a significantly $(p<0.05)$ higher prevalence than urban cats $(29 \%)$. In contrast, the investigative district or the appearance of feces showed no significant difference in the positive rate. Differences in prevalence between the cats originated in private cat owner and in pet shop/breeding cattery and between males and females were also not significant. The breeds such as Persian (71\%), Russian Blue $(60 \%)$, Norwegian Forest cat (100\%), Main Coon (100\%) and American Curl $(100 \%)$ revealed high prevalence of Giardia infections, although the number of animals examined was limited. When comparing the prevalence of Japanese domestic cat to that of pure breeds, there was no significant difference between them.

The present study suggested that $G$. intestinalis infection would be widely spread in household cats of Tohoku district, since the high positive rates for $G$. intestinalis antigen were recorded in three different prefectures of Tohoku. Saito et al. [13-15] previously reported the lower detection rates of $G$. intestinalis such as $0.8-1.7 \%$ in household cats in Hiroshima Prefecture, Japan, based on the results of conventional microscopic fecal examination (concentrated brine flotation technique). This contradiction is probably not due to the investigative district but due to the analyzing method to examine Giardia infection, because the ELISA kit used in 
Table 1. ELISA-positive rates for Giardia intestinalis in household cats of Tohoku district in Japan

\begin{tabular}{|c|c|c|c|}
\hline Profiles of & & ositive rate $(\%)$ & (Positive cats/examined cats) \\
\hline \multicolumn{4}{|l|}{ Prefecture } \\
\hline & Aomori & 42 & $(148 / 353)$ \\
\hline & Akita & 40 & $(75 / 188)$ \\
\hline & Fukushima & 29 & $(17 / 59)$ \\
\hline \multicolumn{4}{|c|}{ Appearance of feces } \\
\hline & Normal & 39 & $(183 / 468)$ \\
\hline & Soft & 39 & $(34 / 88)$ \\
\hline & Diarrhea & 52 & $(23 / 44)$ \\
\hline \multicolumn{4}{|l|}{ Age } \\
\hline & 1-6 months old & $49^{a)}$ & $(70 / 144)$ \\
\hline & 7 months -1 year old & 39 & (77/199) \\
\hline & $2-5$ years old & 39 & $(64 / 165)$ \\
\hline & Over 6 years old & $32^{\mathrm{b})}$ & $(29 / 92)$ \\
\hline \multicolumn{4}{|c|}{ 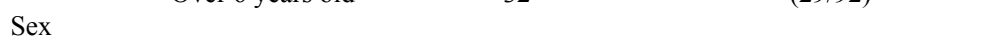 } \\
\hline & Male & 43 & $(104 / 244)$ \\
\hline & Female & 38 & $(136 / 356)$ \\
\hline \multicolumn{4}{|l|}{ Breed } \\
\hline & Japanese domestic cat & 40 & $(220 / 554)$ \\
\hline & Pure breed & 43 & $(20 / 46)$ \\
\hline \multicolumn{4}{|l|}{ Life style } \\
\hline & Indoors & $33^{\mathrm{a}, \mathrm{c})}$ & $(110 / 331)$ \\
\hline & Indoors/outdoors & $45^{\mathrm{b})}$ & $(75 / 166)$ \\
\hline & Outdoors & $53^{\mathrm{d})}$ & $(55 / 103)$ \\
\hline \multicolumn{4}{|c|}{ Environmental condition } \\
\hline & Urban & $29^{\mathrm{a})}$ & $(27 / 92)$ \\
\hline & Suburban & $42^{b)}$ & $(213 / 508)$ \\
\hline \multicolumn{4}{|l|}{ Origin } \\
\hline & Pet shop/breeding cattery & 45 & $(15 / 33)$ \\
\hline & Private cat owner & 40 & $(225 / 567)$ \\
\hline
\end{tabular}

a) VS b) : Significant statistical difference $(\mathrm{p}<0.05)$.

c) VS d) : Significant statistical difference $(\mathrm{p}<0.01)$.

the present study is more sensitive for detecting Giardia infection than the conventional microscopic techniques [4, 8, 16]. McGlade et al. [8] showed that the positive rates for G. intestinalis in 40 feline fecal samples were 5, 80 and $60 \%$ by means of microscopy (zinc sulfate flotation technique), polymerase chain reaction (PCR) and ELISA, respectively.

The detection of $G$. intestinalis antigen showed no relation to fecal appearances in the present study. Furthermore, $90 \%(217 / 240)$ of the cats those were positive for $G$. intestinalis antigen had no diarrhea. Sugano et al. [17] also reported that some of the cats infected with Giardia had no diarrhea. The cats that are positive for Giardia and develop no clinical symptoms will play an important role for the transmission of Giardia as carrier. There was no difference in positive rate between the cats originated from private cat owner and from pet shop/breeding cattery, although the examined number of cats from pet shop/breeding cattery was limited. The previous studies in dogs have been demonstrated that the pet shop/breeding kennel provides the chance for Giardia infection [3-5]. On the other hand, the present study suggests that the pet shop/breeding cattery seems to be not essential for Giardia infection in cats. Further investigation is needed to evaluate the transmission of Giardia in pet shop/breeding cattery, because the sample size from pet shop/breeding cattery was small in the present study.

The positive rate of $G$. intestinalis antigen in cats aged 1 to 6 months old was significantly higher than that in adults over 6 years old. The dogs and cats less than 1 year of age are more likely to be infected with Giardia than older animals because of the immaturity in their immune system [6]. The significantly lower prevalence was recorded in cats kept indoors compared to those kept indoors/outdoors and outdoors. The cats kept indoors will have less opportunity to ingest Giardia cysts from the environment.

Difference in positive rates of $G$. intestinalis antigen between urban cats and suburban cats is probably associated with their life style. Because 78\% (72/92) of urban cats were kept indoors with less chance of Giardia infection, while $51 \%(259 / 508)$ of suburban cats were kept indoors. In dogs, the high percentage infection of $G$. intestinalis is recognized in animals aged 1-6 months old, kept indoors and from pet shop/breeding kennel [3, 4]. In contrast, the high level infection was recorded in cats of all ages, kept indoors/ outdoors and outdoors. Furthermore, the suburban cats showed higher $G$. intestinalis infection. The results of present study suggest that the field contamination with Giardia cysts would play an important role in G. intestinalis 
infection in household cats. Although some of the breeds showed high prevalence, no difference between Japanese domestic cats and pure breeds supports the findings that breed-related resistance or susceptibility to Giardia has been unknown [6].

$G$. intestinalis is divided into several genotypes, and at least 6 genotypes (Assemblage A, B, C, D, E and F) have been isolated from cats $[1,2,7-11,18,19]$. Itagaki et al. [2] recently reported that 3 Giardia isolates from cats in Japan were entirely Assemblage F, which appears to be specific for cats. However, the possibility of zoonotic transmission of $G$. intestinalis from cats to humans will be undeniable, because Assemblage A and B, which have zoonotic potential $[9-12,18,19]$, have been often reported in cats of overseas $[1,11,12,18,19]$. Further studies on genotyping will be necessary to confirm the zoonotic potential of $G$. intestinalis isolated from household cats in Japan.

ACKNOWLEDGMENT. We are grateful to Dr. N. Sasaki (Laboratory of Veterinary Surgery, University of Tokyo, Japan) for critical reading of the manuscript.

\section{REFERENCES}

1. Berrilli, F., Di Cave, D., De Liberato, C., Franco, A., Scaramozzino, P. and Orecchia, P. 2004. Vet. Parasitol. 122: 193199.

2. Itagaki, T., Kinoshita, S., Aoki., M., Itoh, N., Saeki, H., Uetsuki., J., Izumiyama, S., Yagita, K. and Endo, T. 2005. Vet. Parasitol. 133: 283-287.

3. Itoh, N., Muraoka, N., Aoki, M. and Itagaki, T. 2001. J. Jpn. Assoc. Inf. Dis. 75: 671-677.

4. Itoh, N., Muraoka, N., Aoki, M. and Itagaki, T. 2004. J. Jpn
Vet. Med. Assoc. 57: 579-582 (in Japanese with English summary).

5. Itoh, N., Muraoka, N., Saeki, H., Aoki, M. and Itagaki, T. 2005. J. Vet. Med. Sci. 67: 717-718.

6. Kirkpatrick, C. E. 1987. Vet. Clin. North Am. Small Anim. Pract. 17: 1377-1387.

7. Lalle, M., Pozio, E., Capelli, G., Bruschi, F., Crotti, D. and Caccio, S. M. 2004. Int. J. Parasitol. 35: 207-213.

8. McGlade, T. R., Robertson, I. D., Elliot, A. D. and Thompson, R. C. A. 2003. Vet. Parasitol. 110: 197-205.

9. Monis, P. T., Andrews, R. H., Mayrhofer, G. and Ey, P. L. 1999. Mol. Biol. Evol. 16: 1135-1144.

10. Monis, P. T., Andrews, R. H., Mayrhofer, G., Mackrill, J., Kulda, J., Isaac-Renton, J. L. and Ey, P. L. 1998. Parasitology 116: 7-19.

11. Read, C. M., Monis, P. T. and Thompson, R. C. A. 2004. Infect. Genet. Evol. 4: 125-130.

12. Robertson, I. D., Irwin, P. J., Lymbery, A. J. and Thompson, R. C. A. 2000. Int. J. Parasitol. 30: 1369-1377.

13. Saito, T., Hashiguchi, H., Shimatani, K., Miyano, S., Yamaashi, K., Yamaguchi, H., Yoshida, K., Ikeda, F., Kuya, M., Utsunomiya, K. and Tongu, Y. 2004. J. Vet. Med. (Tokyo) 57: 11-14 (in Japanese with English summary).

14. Saito, T., Morishige, K. and Tongu, Y. 1995. Jpn. J. Parasitol. 44: 149-153 (in Japanese with English summary).

15. Saito, T., Yamaguchi, H., Yoshida, K., Kusaura, J., Wada, E., Morishige, K. and Tongu, Y. 1998. J. Vet. Med. (Tokyo) 51: 889-892 (in Japanese with English summary).

16. Schunk, M., Jelinek, T., Wetzel, K. and Nothdurft, H. D. 2001. Eur. J. Clin. Microbiol. Infect. Dis. 20: 389-391.

17. Sugano, H., Yano, A. and Minami, H. 1986. J. Jpn. Vet. Med. Assoc. 39: 504-507 (in Japanese with English summary).

18. Thompson, R. C. A. 2000. Int. J. Parasitol. 30: 1259-1267.

19. Thompson, R. C. A. 2004. Vet. Parasitol. 126: 15-35. 